\title{
The Homotopy Analysis Method in Turning Point Problems
}

Rasha F. Ahmed

Department of Mathematics

College of Computer Science and Mathematics

University of Mosul, Mosul, Iraq
Waleed M. Al-Hayani

waleedalhayani@uomosul.edu.iq

\section{Received on: 06/05/2019}

Accepted on: 27/06/2019

\section{ABSTRACT}

In this paper, we used the homotopy analysis method to ordinary differential equations of type boundary value problems with a parameter representing turning points. To show the high accuracy of the solution results, we compare the numerical results applying the standard homotopy analysis method with the integral equation and the numerical solution of the Simpson and Trapezoidal rules. Also, we give the estimated order of convergence (local) and the global estimated order of convergence along the interval.

Keywords: Homotopy Analysis Method; Turning Points; Boundary Value Problems.

\section{طريقة تحليل هوموتوبي على مسائل نقطة التحول}

$$
\begin{aligned}
& \text { وليل محد الحيانسي } \\
& \text { شا فه أحمد } \\
& \text { قسم الرباضيات } \\
& \text { كلية علوم الحاسوب والرياضيات }
\end{aligned}
$$

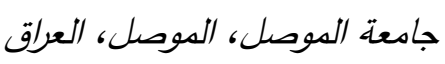

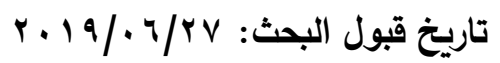

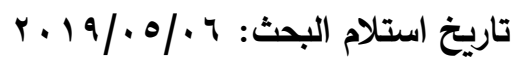

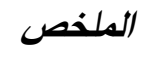

\section{Introduction}

In the 1992, Liao Shijun [3-5] proposed in his $\mathrm{Ph.D}$. dissertation a new and fruitful method (Homotopy Analysis Method (HAM)) for solving linear and nonlinear (ordinary differential, partial differential, integral, etc.) equations. It has been shown that this method yields a rapid convergence of the solutions series to linear and nonlinear deterministic.

In the literature, Al-Hayani and Casasùs [1] applied the Adomian decomposition method (ADM) to the boundary value problems (BVPs) with turning points. Several 
different approaches are currently under study $[6-8,10]$, but we are not aware of other works of this type.

In the consulted bibliography we have not found till now any results of the application of the HAM of differential problems with turning points. For this reason, the principal objective of this article is to apply the HAM for BVPs of ODEs with turning points.

\section{Basic idea of HAM}

Let us consider the following differential equation

$\mathcal{N}[u(x)]=k(x)$,

where $\mathcal{N}$ is a nonlinear operator, $x$ denotes independent variable, $u(x)$ is an unknown function, and $k(x)$ is a known analytic function. For simplicity, we ignore all boundary or initial conditions, which can be treated in the similar way. By means of generalizing the traditional homotopy method, Liao [3-5] constructs the so-called zero-order deformation equation

$(1-q) \mathcal{L}\left[\phi(x ; q)-u_{0}(x)\right]=q h H(x)\{\mathcal{N}[\phi(x ; q)]-k(x)\}$, where $q \in[0,1]$ is an embedding parameter, $h \neq 0$, is a non-zero auxiliary parameter $H(x) \neq 0$ is an auxiliary function, $\mathcal{L}$ is an auxiliary linear operator, $u_{0}(x)$ is an initial guess of $u(x)$ and $\phi(x ; q)$ is an unknown function. It is important to note that one has great freedom to choose auxiliary objects such as $h$ and $\mathcal{L}$ in the HAM. Obviously when $q=0$ and $q=1$, both

$\phi(x ; 0)=u_{0}(x)$ and $\phi(x ; 1)=u(x)$

hold. Thus as $q$ increases from 0 to 1 , the solution $\phi(x ; q)$ varies from the initial guess $u_{0}(x)$ to the solution $u(x)$. Expanding $\phi(x ; q)$ in Taylor series with respect to $\mathrm{q}$, one has

$\phi(x ; q)=u_{0}(x)+\sum_{m=1}^{+\infty} u_{m}(x) q^{m}$,

where

$u_{m}=\left.\frac{1}{m !} \frac{\partial^{m} \phi(x ; q)}{\partial q^{m}}\right|_{q=0}$,

If the auxiliary linear operator, the initial guess, the auxiliary parameter $h$, and the auxiliary function are so properly chosen, then the series (2.4) converges at $q=1$ and one has

$\phi(x ; 1)=u_{0}(x)+\sum_{m=1}^{+\infty} u_{m}(x)$,

which must be one of the original non-linear equation, as proved by Liao [3-5]. If $h=$ -1 , Eq. (2.2) becomes

$(1-q) \mathcal{L}\left[\phi(x ; q)-u_{0}(x)\right]+q\{\mathcal{N}[\phi(x ; q)-k(x)]\}=0$,

which is used mostly in the HPM [2].

According to Eq. (2.5), the governing equations can be deduced from the zeroth-order deformation equations (2.2). We define the vectors

$\overrightarrow{u_{\imath}}=\left\{u_{0}(x), u_{1}(x), \ldots, u_{i}(x)\right\}$.

Differentiating Eq. (2.2) $m$ times with respect to the embedding parameter $q$ and then setting $q=0$ and finally dividing them by $m$ !, we have the so-called $m t h$-order deformation equation

$\mathcal{L}\left[u_{m}(x)-x_{m} u_{m-1}(x)\right]=h \mathcal{R}_{m}\left(\vec{u}_{m-1}\right)$, 
where

$\mathcal{R}_{m}\left(\vec{u}_{m-1}\right)=\left.\frac{1}{(m-1) !} \frac{\partial^{m-1}\{\mathcal{N}[\phi(; q)]-k(x)\}}{\partial q^{m-1}}\right|_{q=0}$,

and

$x_{m}= \begin{cases}0, & m \leq 1 \\ 1, & m>1 .\end{cases}$

It should be emphasized that $u_{m}(x)(m \geq 1)$ are governed by the linear equation (2.8) with the linear boundary conditions that come from the original problem, which can be easily solved by symbolic computation softwares such as Maple and Mathematica.

\section{HAM Applied to BVPs of large parameter}

We consider the BVP $[1,9]$ :

$u^{\prime \prime}+\lambda^{2} q(x) u=0$,

$u(-1)=-1, u(1)=1$,

where $\lambda$ is a real constant and $q(x)$ is a function. The exact solution is not given here. The solution using WKB method is given by [9]

$u_{W K B}(x)=\frac{c_{1} \cos \left(\lambda \int \sqrt{q(x)} d x\right)+c_{2} \sin \left(\lambda \int \sqrt{q(x)} d x\right)}{\sqrt[4]{q(x)}}$.

To solve Eq. (3.1) by means of the standard HAM, we choose the initial approximation $u_{0}(x)=c_{1}+c_{2} x$,

and the linear operator

$\mathcal{L}[\phi(x ; q)]=\frac{\partial^{2} \phi(x ; q)}{\partial x^{2}}$

with the property

$\mathcal{L}\left[c_{1}+c_{2} x\right]=0$,

where $c_{1}, c_{2}$ are constants of integration calculated from the boundary conditions (3.2).

Furthermore, Eq. (3.1) suggests that we define the nonlinear operator as

$\mathcal{N}[\phi(x ; q)]=\frac{\partial^{2} \phi(x ; q)}{\partial x^{2}}+\lambda^{2} q(x) \phi(x ; q)$,

Using the above definition, we construct the zeroth-order deformation equation as in (2.2) and (2.3) and the $m t h$-order deformation equation for $m \geq 1$ is

$\mathcal{L}\left[u_{m}(x)-x_{m} u_{m-1}(x)\right]=h R_{m}\left(\vec{u}_{m-1}\right)$,

with the boundary conditions

$u_{m}(-1)=0, \quad u_{m}(1)=0$,

where

$R_{m}\left(\vec{u}_{m-1}\right)=u_{m-1}^{\prime \prime}+\lambda^{2} q(x) u_{m-1}$,

Now, the solution of the $m t h$-order deformation equation (3.7) for $m \geq 1$ is

$u_{m}(x)=x_{m} y_{m-1}(x)+h \int_{0}^{x} \int_{0}^{x} R_{m}\left(\vec{u}_{m-1}\right) d x d x$,

We give the results of our approximation HAM with the WKB solution (3.3) in the following figures (Figs.1-20 ). In order to give an idea of the accuracy of the method, we also give the approximation HAM $(n=20)$ with some approximations of lower order.

We symbolize the continuous line for the HAM $(n=20)$ and the symbol $\diamond$ for WKB and the $\operatorname{HAM}(n=5,6,19)$.

Case 1. Taking $q(x)=x$.

From Eq. (3.9) the first iterations are given by: 
$u_{0}(x)=c_{1}+c_{2} x$,

$u_{1}(x)=\frac{1}{12} h \lambda^{2} x^{3}\left(2 c_{1}+c_{2} x\right)$,

$u_{2}(x)=\frac{1}{6} c_{1} h(1+h) \lambda^{2} x^{3}+\frac{1}{12} c_{2} h(1+h) \lambda^{2} x^{4}+\frac{1}{180} c_{1} h^{2} \lambda^{4} x^{6}+\frac{1}{504} c_{2} h^{2} \lambda^{4} x^{7}$,

etc., in the same manner the rest of the iterations. Thus, the solution in a series form when $\boldsymbol{h}=\mathbf{- 1}$ is

$u(x)=u_{0}(x)+\sum_{m=1}^{20} u_{m}(x)$

In Table 1 display a comparison of the numerical results applying the HAM $(\boldsymbol{m}=20$ ), Iteration of the Integral Equation (IIE) (3.9), and the numerical solution of (3.9), with the Simpson rule (SIMP) and the trapezoidal rule (TRAP). Twenty points have been used in the Simpson and trapezoidal rules. The estimated orders of convergence (EOC) are given in Table 2, and the global estimated orders of convergence (GEOC) within the interval $[-1,1]$ are given in Table 3. See also Figs. 1-6.

Table 1. Numerical results for the case 1 for $\lambda=20$ with step size 0.05 .

\begin{tabular}{ccccc}
\hline $\boldsymbol{x}$ & HAM & IIE & TRAP & SIMP \\
\hline-1.0 & -0.999999999 & -0.999690995 & -0.999495025 & -0.999688272 \\
-0.8 & -0.023834292 & -0.023834292 & -0.023834291 & -0.023834292 \\
-0.6 & 0.000236321 & 0.000236321 & 0.000236321 & 0.000236321 \\
-0.4 & 0.021131965 & 0.021131965 & 0.021131965 & 0.021131965 \\
-0.2 & 0.217401482 & 0.217401482 & 0.217401482 & 0.217401482 \\
0.0 & 1.038054523 & 1.038054523 & 1.038054523 & 1.038054523 \\
0.2 & 1.380574788 & 1.380574788 & 1.380574788 & 1.380574788 \\
0.4 & -1.054531820 & -1.054531820 & -1.054531820 & -1.054531820 \\
0.6 & 0.721696550 & 0.721696550 & 0.721696550 & 0.721696550 \\
0.8 & -0.824992723 & -0.824992721 & -0.824992721 & -0.824992721 \\
1.0 & 1.000000000 & 1.000863433 & 1.001431522 & 1.000871665 \\
\hline
\end{tabular}

Table 2. EOC for the case 1.

\begin{tabular}{cccccccc}
\hline$\lambda$ & $x=-0.8$ & $x=-0.6$ & $x=-0.4$ & $x= \pm 0.2$ & $x=0.4$ & $x=0.6$ & $x=0.8$ \\
\hline 15 & 1.1937 & 1.1459 & 1.1870 & 1.1873 & 1.1873 & 1.1873 & 1.1872 \\
20 & 1.4629 & 1.2654 & 1.5955 & 1.5956 & 1.5956 & 1.5956 & 1.5954 \\
\hline
\end{tabular}

Table 3. GEOC for the case 1.

\begin{tabular}{lcccc}
\hline$\lambda$ & 5 & 10 & 15 & 20 \\
\hline GEOC & 1.0356 & 1.0409 & 1.1873 & 1.5957 \\
\hline
\end{tabular}




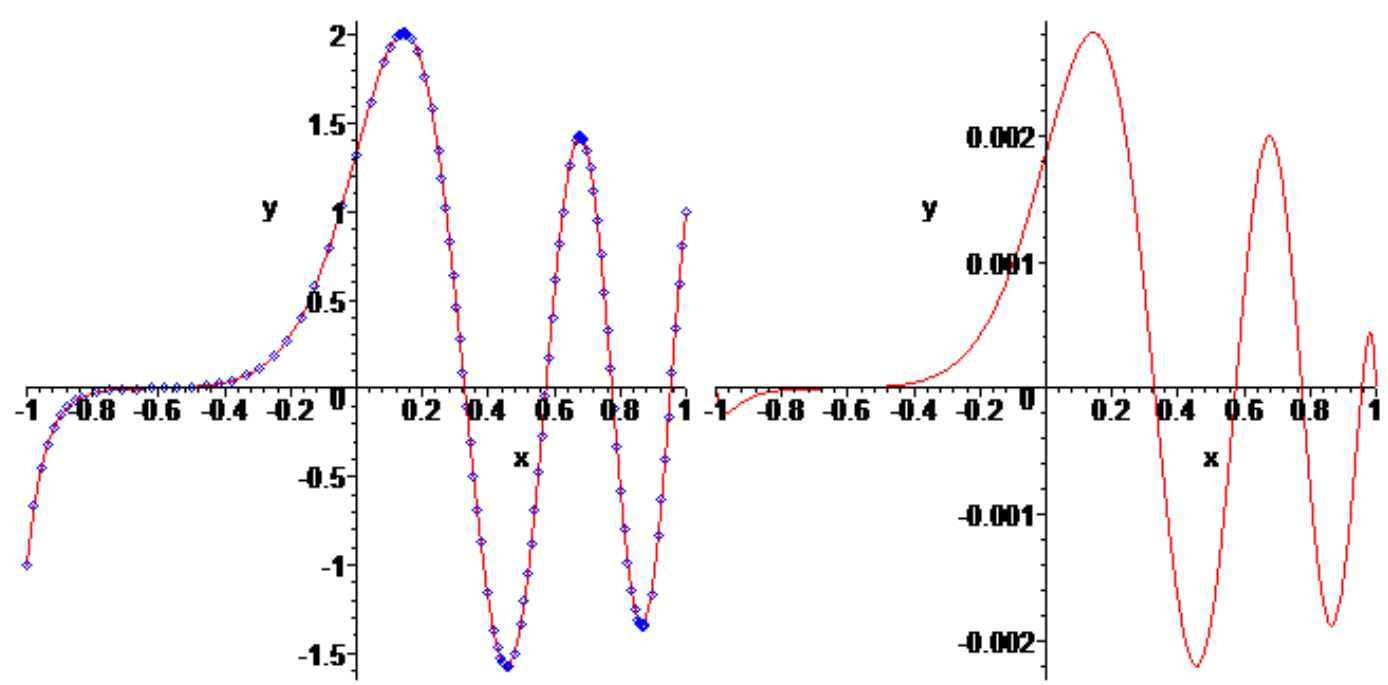

Fig. 1. $\mathrm{HAM}_{\mathrm{m}=20}, \mathrm{HAM}_{\mathrm{m}=19}, \lambda=19$

Fig. 2. $\mathrm{HAM}_{\mathrm{m}=20}-\mathrm{HAM}_{\mathrm{m}=19}, \lambda=19$
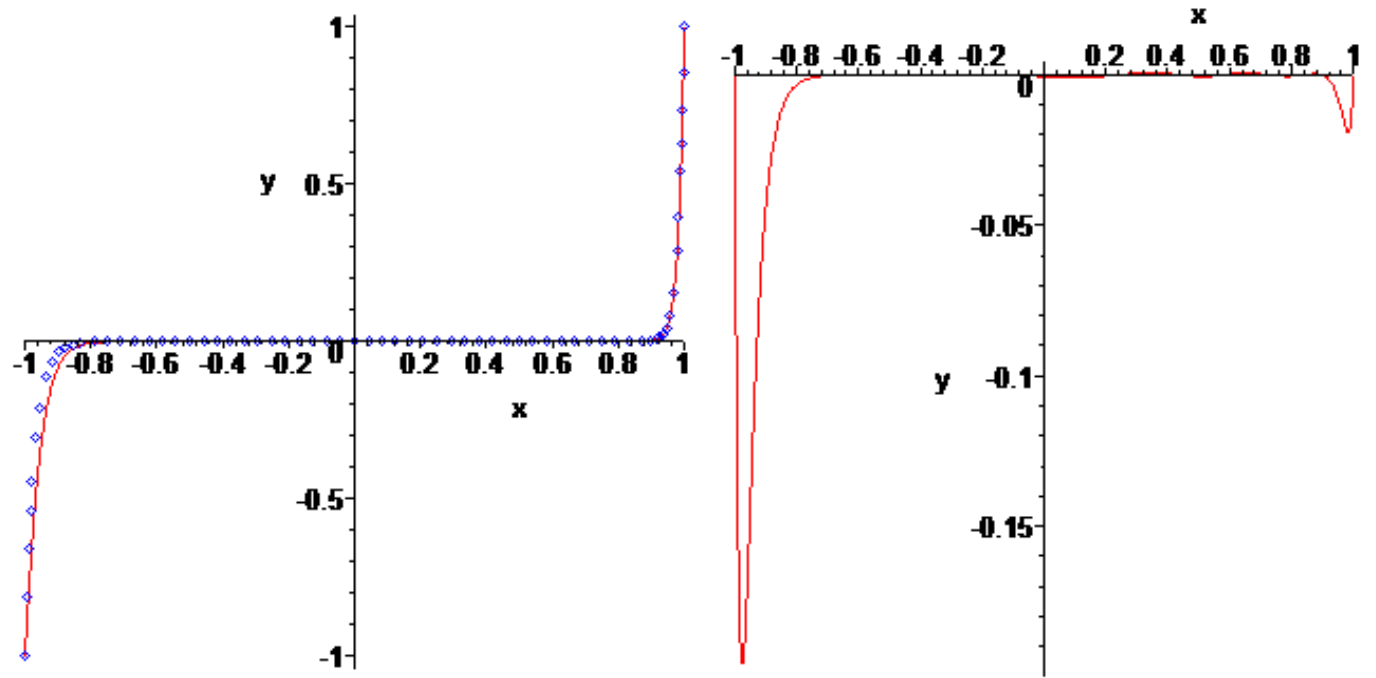

Fig. 3. $\mathrm{HAM}_{\mathrm{m}=20}, \mathrm{HAM}_{\mathrm{m}=19}, \lambda=29$

Fig. 4. $\mathrm{HAM}_{\mathrm{m}=20}-\mathrm{HAM}_{\mathrm{m}=19}, \lambda=29$

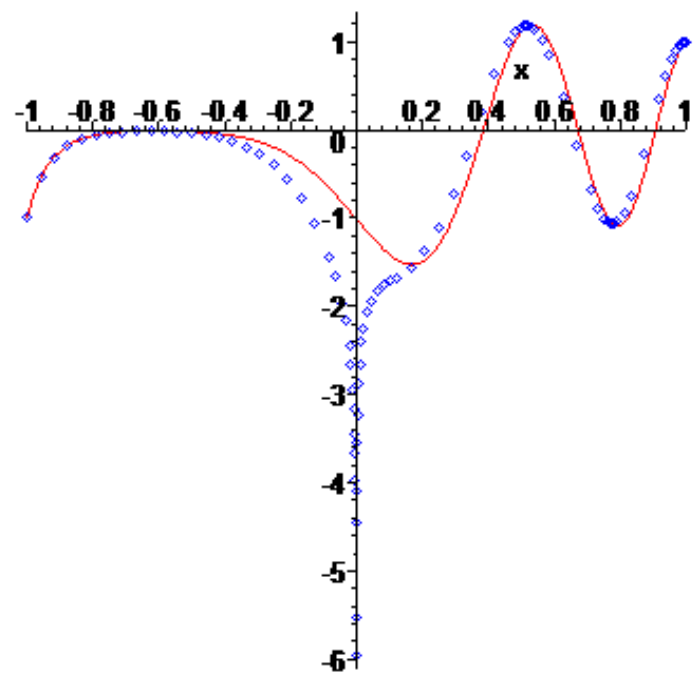

Fig. 5. $\mathrm{WKB}, \mathrm{HAM}_{\mathrm{m}=20}, \lambda=14.95$

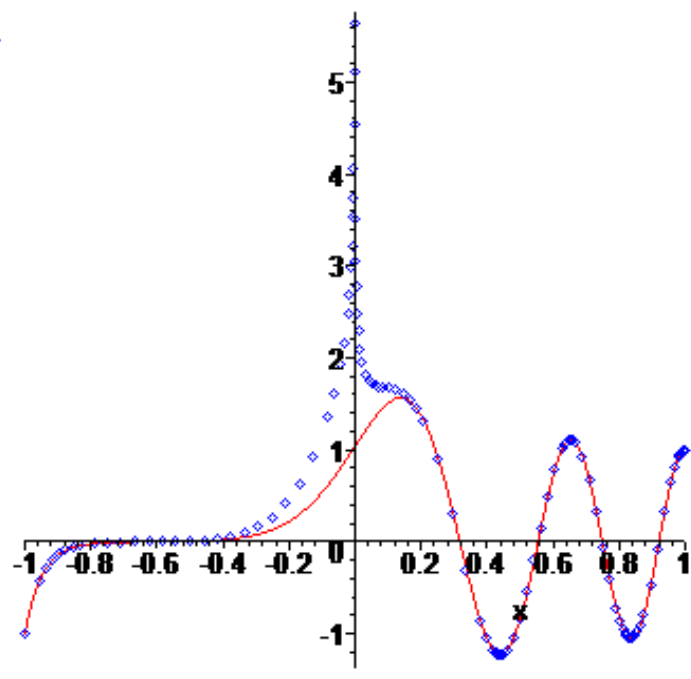

Fig. 6. $\mathrm{WKB}, \mathrm{HAM}_{\mathrm{m}=20}, \lambda=19.95$ 
Case 2. Taking $q(x)=x^{2}$.

From Eq. (3.9) the first iterations are given by:

$u_{0}(x)=c_{1}+c_{2} x$,

$u_{1}(x)=\frac{1}{60} h \lambda^{2} x^{4}\left(5 c_{1}+3 c_{2} x\right)$,

$u_{2}(x)=\frac{1}{12} c_{1} h(1+h) \lambda^{2} x^{4}+\frac{1}{20} c_{2} h(1+h) \lambda^{2} x^{5}+\frac{1}{672} c_{1} h^{2} \lambda^{4} x^{8}+\frac{1}{1440} c_{2} h^{2} \lambda^{4} x^{9}$, $\vdots$

etc., in the same manner the rest of the iterations. Thus, the solution in a series form when $\boldsymbol{h}=-\mathbf{1}$ is

$u(x)=u_{0}(x)+\sum_{m=1}^{20} u_{m}(x)$

In Table 4 display a comparison of the numerical results applying the HAM ( $\boldsymbol{m}=$ 20), Iteration of the Integral Equation (IIE) (3.9), and the numerical solution of (3.9), with the Simpson rule (SIMP) and the trapezoidal rule (TRAP). Twenty points have been used in the Simpson and trapezoidal rules. The EOC are given in Table 5, and the GEOC within the interval $[-1,1]$ are given in Table 6. See also Figs. 7-10.

Table 4. Numerical results for the case 2 for $\lambda=5$ with step size 0.05 .

\begin{tabular}{ccccc}
\hline $\boldsymbol{x}$ & HAM & IIE & TRAP & SIMP \\
\hline-1.0 & -0.999999999 & -0.999999999 & -0.999999999 & -0.999999999 \\
-0.8 & -3.692707985 & -3.692707985 & -3.692707985 & -3.692707985 \\
-0.6 & -4.208172135 & -4.208172135 & -4.208172135 & -4.208172135 \\
-0.4 & -3.214221610 & -3.214221610 & -3.214221610 & -3.214221610 \\
-0.2 & -1.656435055 & -1.656435055 & -1.656435055 & -1.656435055 \\
0.0 & 0.000000000 & 0.000000000 & 0.000000000 & 0.000000000 \\
0.2 & 1.656435055 & 1.656435055 & 1.656435055 & 1.656435055 \\
0.4 & 3.214221610 & 3.214221610 & 3.214221610 & 3.214221610 \\
0.6 & 4.208172135 & 4.208172135 & 4.208172135 & 4.208172135 \\
0.8 & 3.692707985 & 3.692707985 & 3.692707985 & 3.692707985 \\
1.0 & 0.999999999 & 0.999999999 & 0.999999999 & 0.999999999 \\
\hline
\end{tabular}

Table 5. EOC for the case 2

\begin{tabular}{rcccc}
\hline$\lambda$ & $x= \pm 0.2$ & $x= \pm 0.4$ & $x= \pm 0.6$ & $x= \pm 0.8$ \\
\hline 20 & 1.1814 & 1.1814 & 1.1814 & 1.1814 \\
25 & 1.3668 & 1.3668 & 1.3668 & 1.3668 \\
\hline
\end{tabular}

Table 6. GEOC for the case 2

\begin{tabular}{lccccc}
\hline$\lambda$ & 5 & 10 & 15 & 20 & 25 \\
\hline GEOC & 1.0306 & 1.0433 & 1.0599 & 1.1814 & 1.3668 \\
\hline
\end{tabular}




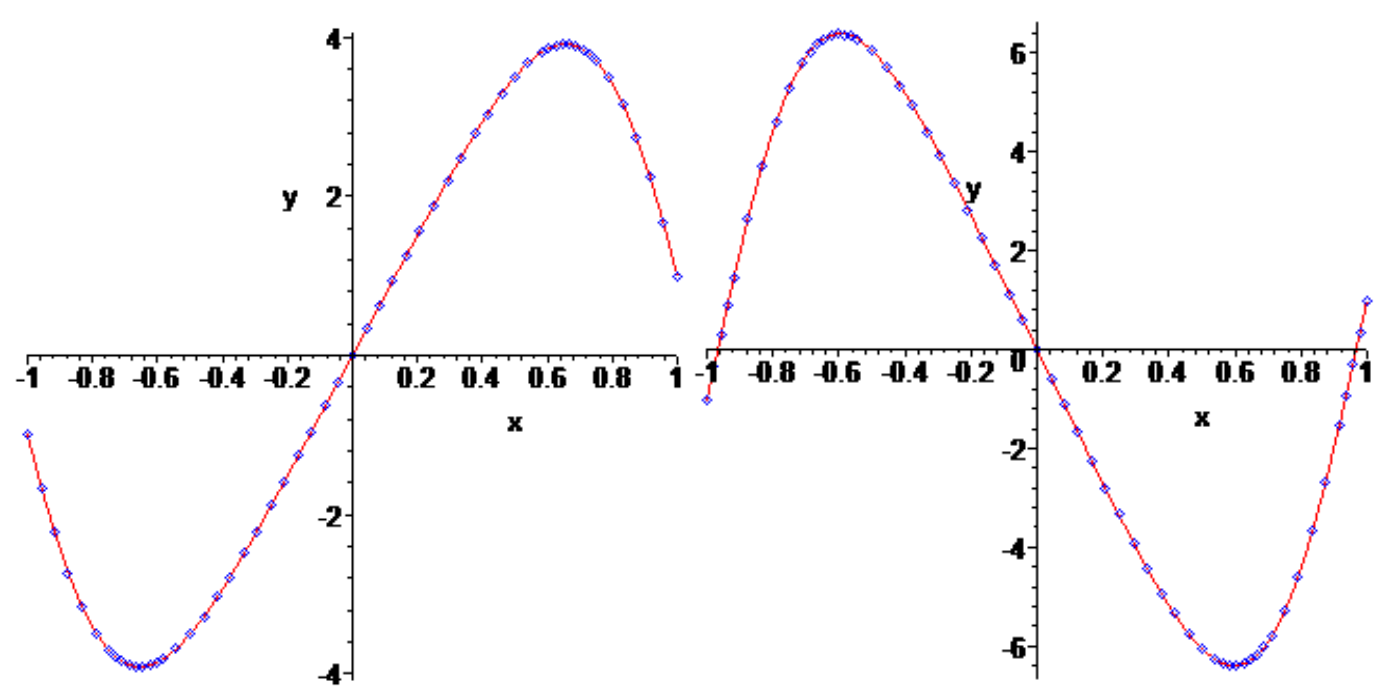

Fig. 7. $\mathrm{HAM}_{\mathrm{m}=20}, \mathrm{HAM}_{\mathrm{m}=6}, \lambda=4.95$

Fig. 8. $\mathrm{HAM}_{\mathrm{m}=20}, \mathrm{HAM}_{\mathrm{m}=6}, \lambda=5.95$

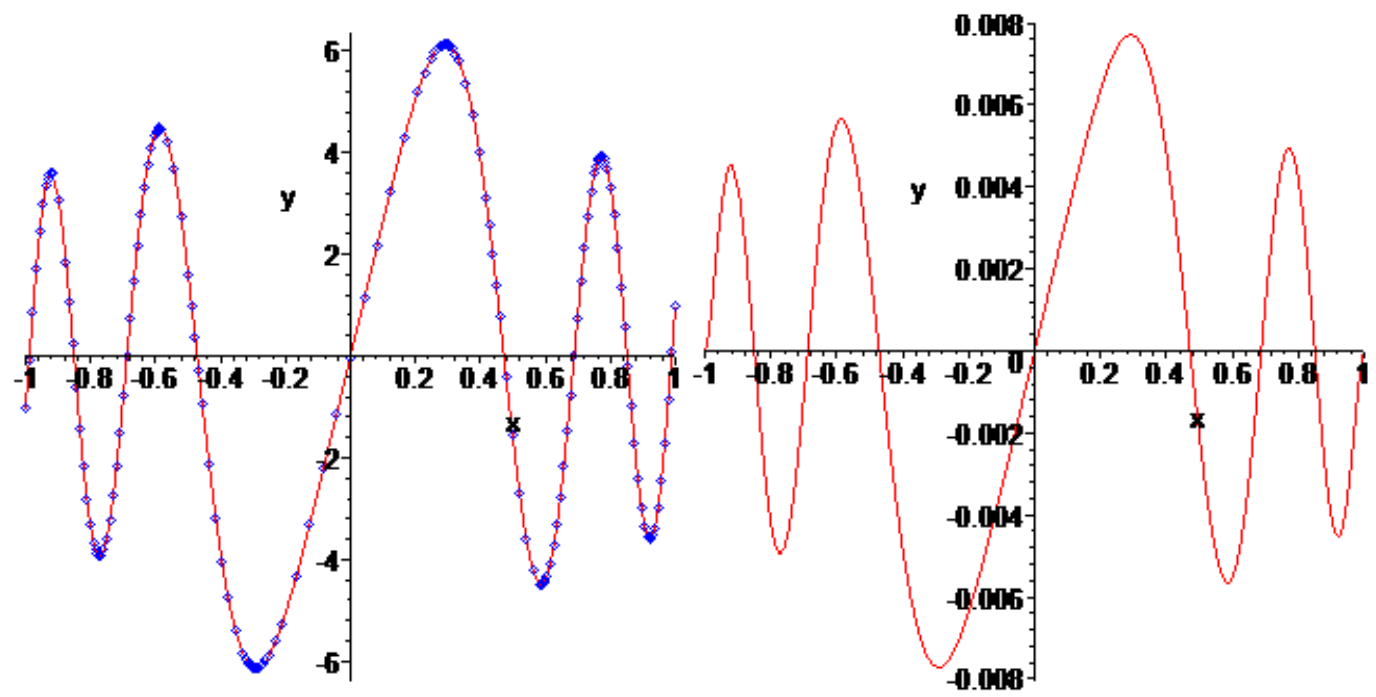

Fig. 9. $\mathrm{HAM}_{\mathrm{m}=20}, \mathrm{HAM}_{\mathrm{m}=19}, \lambda=24.5$

Fig. 10. $\mathrm{HAM}_{\mathrm{m}=20}-\mathrm{HAM}_{\mathrm{m}=19}, \lambda=24.5$

Case 3. Taking $q(x)=x^{3}$.

From Eq. (3.9) the first iterations are given by:

$u_{0}(x)=c_{1}+c_{2} x$,

$u_{1}(x)=\frac{1}{60} h \lambda^{2} x^{5}\left(3 c_{1}+2 c_{2} x\right)$,

$u_{2}(x)=\frac{1}{20} c_{1} h(1+h) \lambda^{2} x^{5}+\frac{1}{30} c_{2} h(1+h) \lambda^{2} x^{6}+\frac{1}{1800} c_{1} h^{2} \lambda^{4} x^{10}$

$+\frac{1}{3300} c_{2} h^{2} \lambda^{4} x^{11}$

etc., in the same manner the rest of the iterations. Thus, the solution in a series form when $\boldsymbol{h}=-\mathbf{1}$ is

$u(x)=u_{0}(x)+\sum_{m=1}^{20} u_{m}(x)$ 
In Table 7 display a comparison of the numerical results applying the HAM ( $\boldsymbol{m}=$ 20), Iteration of the Integral Equation (IIE) (3.9), and the numerical solution of (3.9), with the Simpson rule (SIMP) and the trapezoidal rule (TRAP). Twenty points have been used in the Simpson and trapezoidal rules. The EOC are given in Table 8, and the GEOC within the interval $[-1,1]$ are given in Table 9. See also Figs. 11-14.

Table 7. Numerical results for the case 3 for $\lambda=5$ with step size 0.05 .

\begin{tabular}{ccccc}
\hline $\boldsymbol{x}$ & HAM & IIE & TRAP & SIMP \\
\hline-1.0 & -0.999999999 & -0.999999999 & -0.999999999 & -0.999999999 \\
-0.8 & -0.320439009 & -0.320439009 & -0.320439009 & -0.320439009 \\
-0.6 & 0.147289780 & 0.147289780 & 0.147289780 & 0.147289780 \\
-0.4 & 0.632022774 & 0.632022774 & 0.632022774 & 0.632022774 \\
-0.2 & 1.154174135 & 1.154174135 & 1.154174135 & 1.154174135 \\
0.0 & 1.687787916 & 1.687787916 & 1.687787916 & 1.687787916 \\
0.2 & 2.221116940 & 2.221116940 & 2.221116940 & 2.221116940 \\
0.4 & 2.725443713 & 2.725443713 & 2.725443713 & 2.725443713 \\
0.6 & 3.027667586 & 3.027667586 & 3.027667586 & 3.027667586 \\
0.8 & 2.652143539 & 2.652143539 & 2.652143539 & 2.652143539 \\
1.0 & 0.999999999 & 0.9999999999 & 0.9999999999 & 0.9999999999 \\
\hline
\end{tabular}

Table 8. EOC for the case 3

\begin{tabular}{cccccc}
\hline$\lambda$ & $x=-0.6$ & $x=-0.4$ & $x= \pm 0.2$ & $x=0.4$ & $x=0.6$ \\
\hline 20 & 1.0995 & 1.0993 & 1.0993 & 1.0993 & 1.0993 \\
25 & 1.1841 & 1.1845 & 1.1846 & 1.1846 & 1.1846 \\
30 & 1.4476 & 1.4476 & 1.4476 & 1.4476 & 1.4476 \\
\hline
\end{tabular}

Table 9. GEOC for the case 3

\begin{tabular}{lcccccc}
\hline$\lambda$ & 5 & 10 & 15 & 20 & 25 & 30 \\
\hline GEOC & 1.0286 & 1.0388 & 1.0466 & 1.0993 & 1.1846 & 1.4476 \\
\hline
\end{tabular}

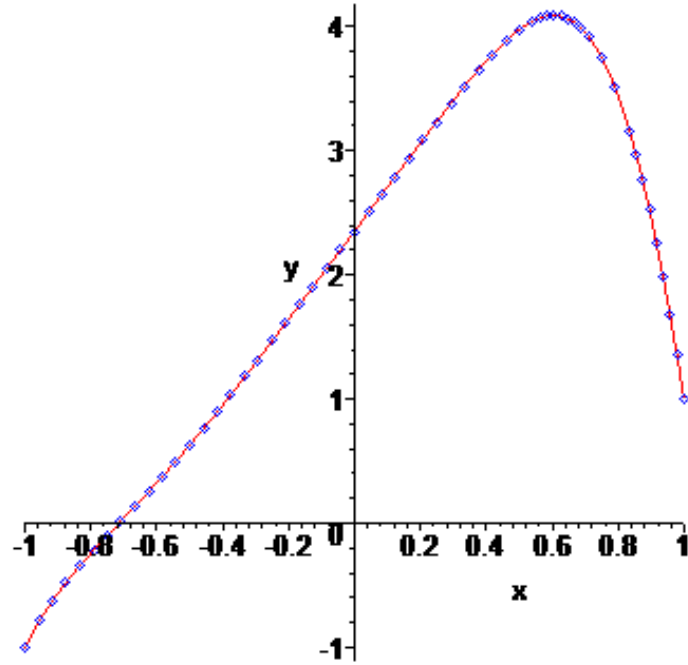

Fig. 11. $\mathrm{HAM}_{\mathrm{m}=20}, \mathrm{HAM}_{\mathrm{m}=6}, \lambda=5.25$

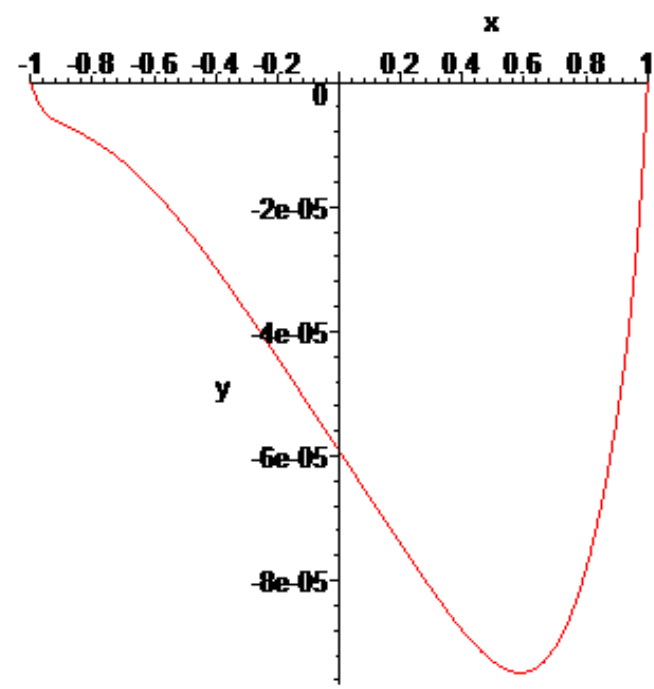

Fig. 12. $\mathrm{HAM}_{\mathrm{m}=20}-\mathrm{HAM}_{\mathrm{m}=6}, \lambda=5.25$ 


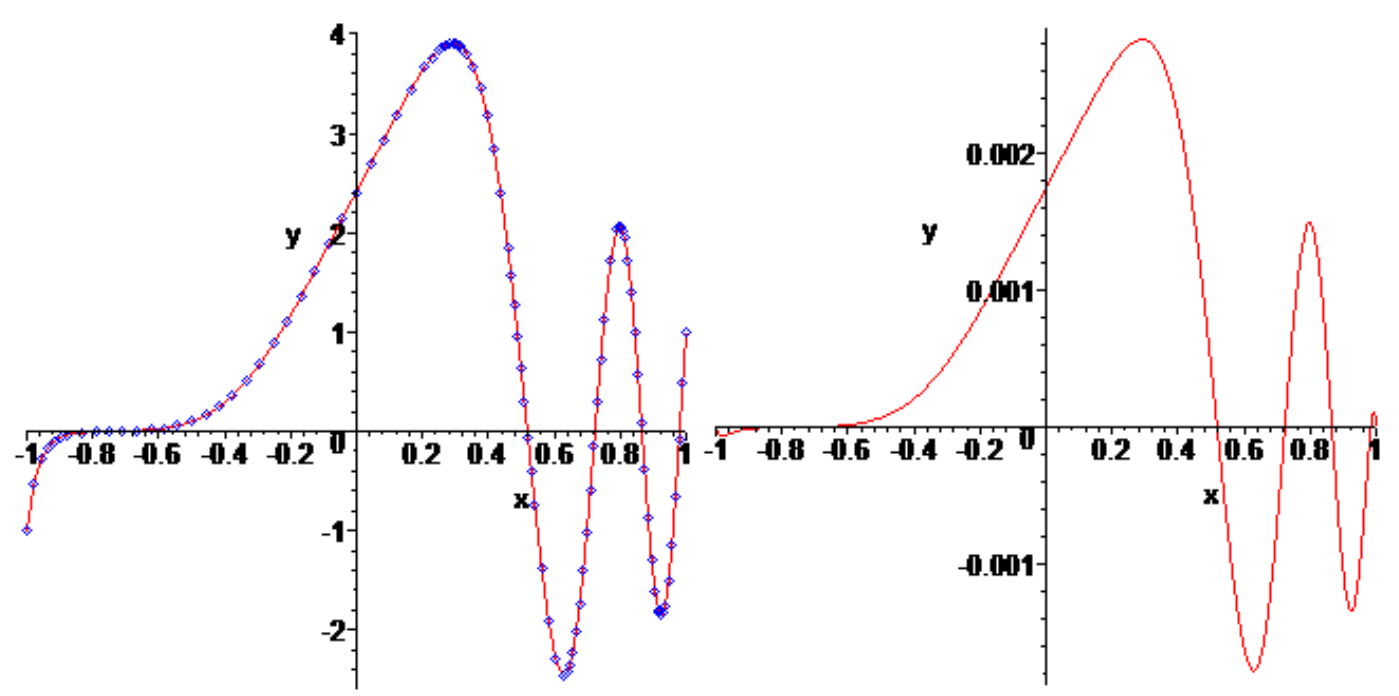

Fig. 13. $\mathrm{HAM}_{\mathrm{m}=20}, \mathrm{HAM}_{\mathrm{m}=19}, \lambda=31$

Fig. 14. $\mathrm{HAM}_{\mathrm{m}=20}-\mathrm{HAM}_{\mathrm{m}=19}, \lambda=31$

Case 4. Taking $q(x)=\left(x-\frac{1}{2}\right)\left(x+\frac{1}{2}\right)$.

From Eq. (3.9) the first iterations are given by:

$u_{0}(x)=c_{1}+c_{2} x$,

$u_{1}(x)=-\frac{1}{8} c_{1} h \lambda^{2} x^{2}-\frac{1}{24} c_{2} h \lambda^{2} x^{3}+\frac{1}{12} c_{1} h \lambda^{2} x^{4}+\frac{1}{20} c_{2} h \lambda^{2} x^{5}$,

$\vdots$

etc., in the same manner the rest of the iterations. Thus, the solution in a series form when $\boldsymbol{h}=-\mathbf{1}$ is

$u(x)=u_{0}(x)+\sum_{m=1}^{20} u_{m}(x)$

In Table 10 display a comparison of the numerical results applying the HAM ( $\boldsymbol{m}=$ 20), Iteration of the Integral Equation (IIE) (3.9), and the numerical solution of (3.9), with the Simpson rule (SIMP) and the trapezoidal rule (TRAP). Twenty points have been used in the Simpson and trapezoidal rules. The EOC are given in Table 11, and the GEOC within the interval $[-1,1]$ are given in Table 12. See also Figs. 15-20.

Table 10. Numerical results for the case 4 for $\lambda=5$ with step size 0.05 .

\begin{tabular}{ccccc}
\hline $\boldsymbol{x}$ & HAM & IIE & TRAP & SIMP \\
\hline-1.0 & -0.999999999 & -0.999999999 & -0.999999999 & -0.999999999 \\
-0.8 & -1.227398073 & -1.227398073 & -1.227398073 & -1.227398073 \\
-0.6 & -0.981531405 & -0.981531405 & -0.981531405 & -0.981531405 \\
-0.4 & -0.608911883 & -0.608911883 & -0.608911883 & -0.608911883 \\
-0.2 & -0.277766349 & -0.277766349 & -0.277766349 & -0.277766349 \\
0.0 & 0.000000000 & 0.000000000 & 0.000000000 & 0.000000000 \\
0.2 & 0.277766349 & 0.277766349 & 0.277766349 & 0.277766349 \\
0.4 & 0.608911883 & 0.608911883 & 0.608911883 & 0.608911883 \\
0.6 & 0.981531405 & 0.981531405 & 0.981531405 & 0.981531405
\end{tabular}




\begin{tabular}{lllll}
0.8 & 1.227398073 & 1.227398073 & 1.227398073 & 1.227398073 \\
1.0 & 0.999999999 & 0.999999999 & 0.999999999 & 0.999999999 \\
\hline
\end{tabular}

Table 11. EOC for the case 4

\begin{tabular}{ccccc}
\hline$\lambda$ & $x= \pm 0.2$ & $x= \pm 0.4$ & $x= \pm 0.6$ & $x= \pm 0.8$ \\
\hline 25 & 1.0499 & 1.0499 & 1.0499 & 1.0501 \\
30 & 1.0300 & 1.0301 & 1.0301 & 1.0326 \\
35 & 1.1177 & 1.1177 & 1.1177 & 1.1179 \\
40 & 1.1403 & 1.1403 & 1.1403 & 1.1404 \\
\hline
\end{tabular}

Table 12. GEOC for the case 4

\begin{tabular}{lcccccccc}
\hline$\lambda$ & 5 & 10 & 15 & 20 & 25 & 30 & 35 & 40 \\
\hline GEOC & 1.0269 & 1.0348 & 1.0415 & 1.0449 & 1.0531 & 1.0711 & 1.1206 & 1.1435 \\
\hline
\end{tabular}

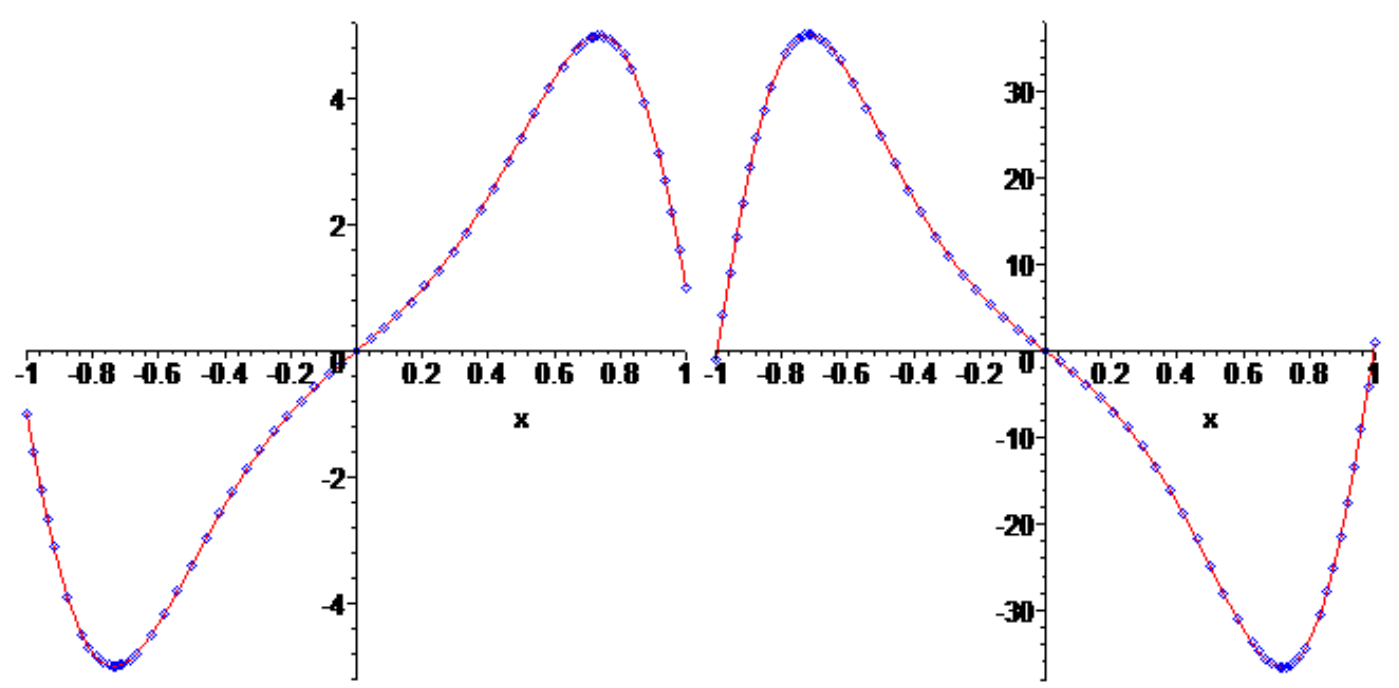

Fig. 15. $\mathrm{HAM}_{\mathrm{m}=20}, \mathrm{HAM}_{\mathrm{m}=5}, \lambda=8$

Fig. 16. . $\mathrm{HAM}_{\mathrm{m}=20}, \mathrm{HAM}_{\mathrm{m}=6}, \lambda=9$

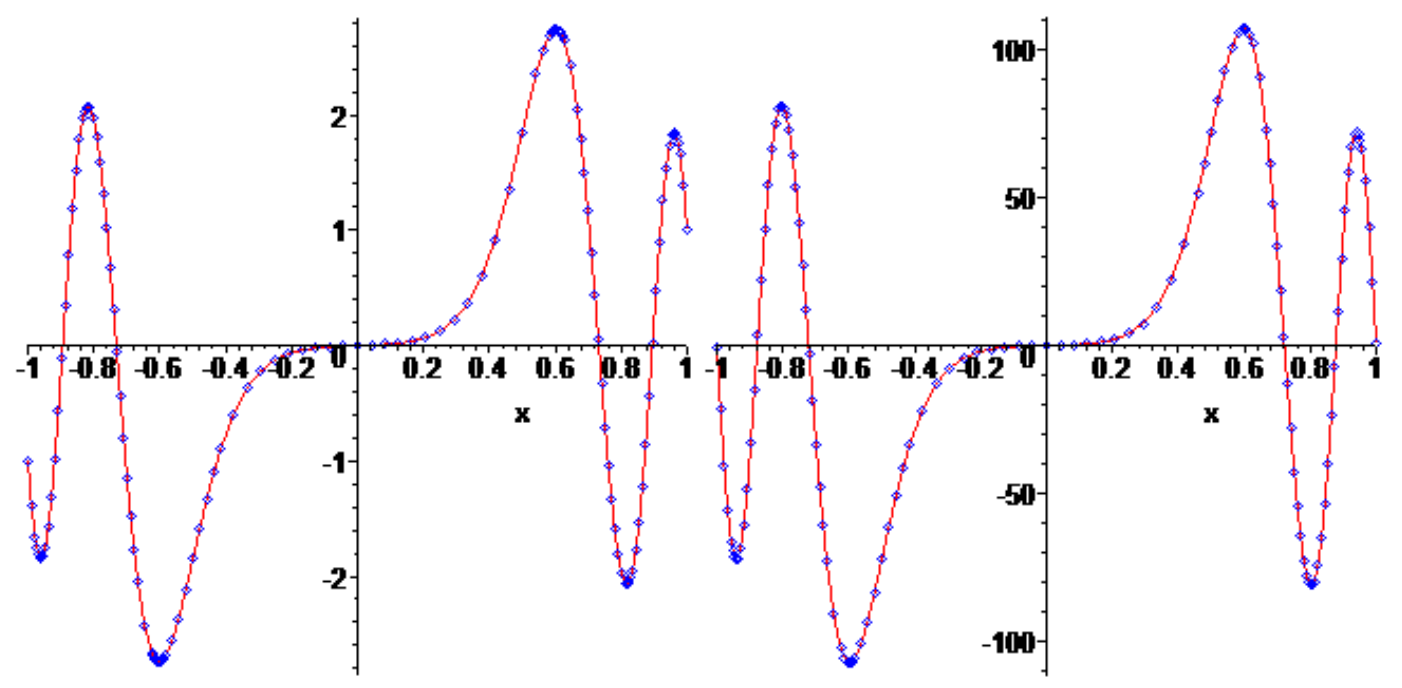

Fig. 17. $\mathrm{HAM}_{\mathrm{m}=20}, \mathrm{HAM}_{\mathrm{m}=19}, \lambda=30$

Fig. 18. $\mathrm{HAM}_{\mathrm{m}=20}, \mathrm{HAM}_{\mathrm{m}=19}, \lambda=32.15$ 


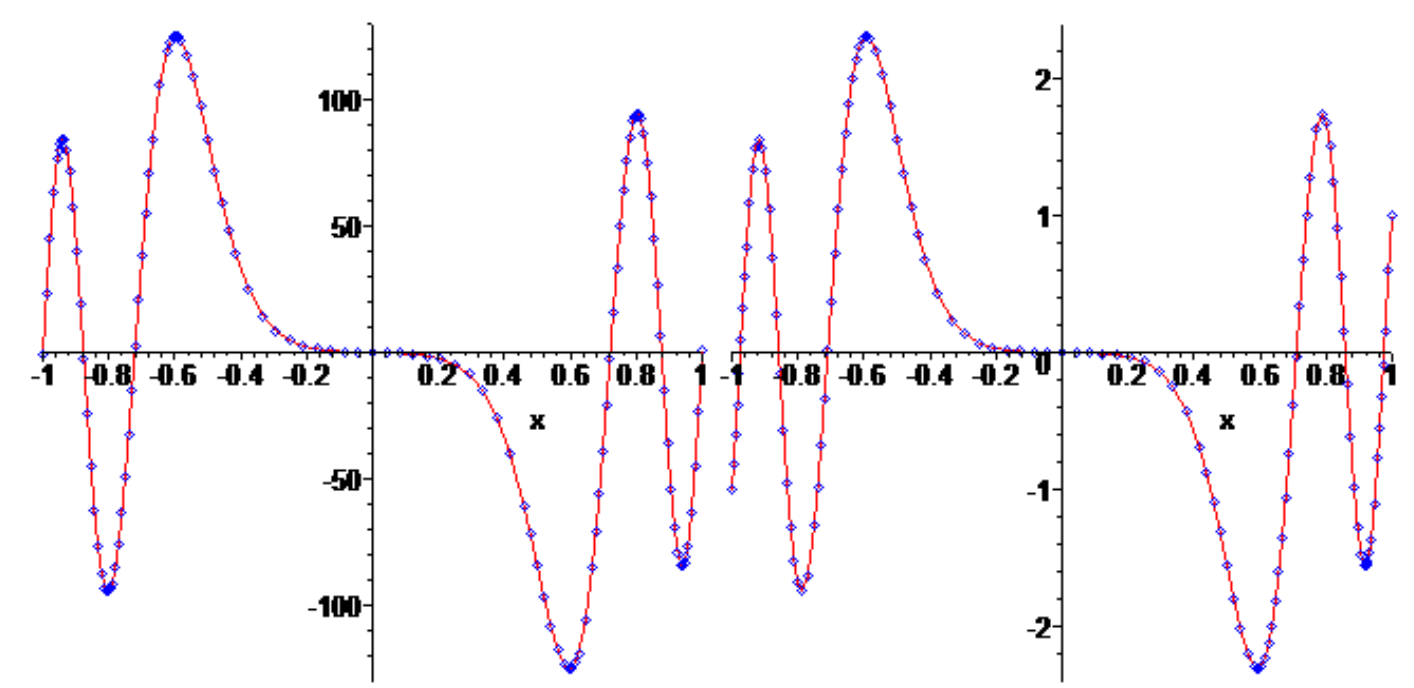

Fig. 19. $\mathrm{HAM}_{\mathrm{m}=20}, \mathrm{HAM}_{\mathrm{m}=19}, \lambda=32.25$ Fig. 20. $\mathrm{HAM}_{\mathrm{m}=20}, \mathrm{HAM}_{\mathrm{m}=19}, \lambda=35$

\section{HAM Applied to BVPs of small parameter}

Consider the following BVP for a non-homogeneous equation [8]

$\varepsilon u^{\prime \prime}+2 u^{\prime}=-u-0.3, \quad 0<\varepsilon \ll 1$,

$u(-1)=0.1, u(1)=0.2$.

The exact solution of (4.1), (4.2) is

$u_{\text {Exact }}(x)=0.1\left[\frac{\left(4 e^{-m_{2}}-5 e^{m_{2}}\right) e^{-m_{1} x}-\left(4 e^{-m_{1}}-5 e^{m_{1}}\right) e^{-m_{2} x}}{e^{m_{1}-m_{2}-} e^{-\left(m_{1}-m_{2}\right)}}\right]-0.3$,

where

$m_{1,2}=\frac{(1 \mp \sqrt{1-\varepsilon})}{\varepsilon}$.

Then to solve (4.1) and (4.2) by means of standard HAM, we choose the initial approximation

$u_{0}(x)=\varepsilon \frac{2 e^{40}-e^{20-20 \varepsilon}-1}{e^{40}-1}$,

and the linear operator

$\mathcal{L}[\phi(x ; q)]=\varepsilon \frac{\partial^{2} \phi(x ; q)}{\partial x^{2}}+2 \frac{\partial \phi(x ; q)}{\partial x}$,

with the property

$\mathcal{L}\left[c_{1}+c_{2} e^{-\frac{2 x}{\varepsilon}}\right]=0$,

where $c_{1}, c_{2}$ are constants of integration. Furthermore, equation (4.1) suggests that we define the non-linear operator as

$\mathcal{N}[\phi(x ; q)]=\varepsilon \frac{\partial^{2} \phi(x ; q)}{\partial x^{2}}+2 \frac{\partial \phi(x ; q)}{\partial x}+\phi(x ; q)+0.3$

Using the above definition, we construct the zeroth-order deformation equation as in (2.2) and (2.3) and the $m t h$-order deformation equation for $m \geq 1$ is

$\mathcal{L}\left[u_{m}(x)-x_{m} u_{m-1}(x)\right]=h G(x, \xi) R_{m}\left(\vec{u}_{m-1}\right)$,

with the boundary conditions

$u_{m}(-1)=0, \quad u_{m}(1)=0$,

where $G(x, \xi)$ is the Green's function given by 
$G(x, \xi)= \begin{cases}-\frac{1}{2} \frac{\left[e^{\left(\frac{(-2-2 x)}{\varepsilon}\right)}-1\right]\left[e^{\left(\frac{(2-2 \xi)}{\varepsilon}\right)}-1\right]}{e^{\left(\frac{(-2-2 \xi)}{\varepsilon}\right)}-e^{\left(\frac{(2-2 \xi)}{\varepsilon}\right)},} & -1 \leq x \leq \xi \leq 1, \\ -\frac{1}{2} \frac{\left[e^{\left(\frac{(-2-2 \xi)}{\varepsilon}\right)}-1\right]\left[e^{\left(\frac{(2-2 x)}{\varepsilon}\right)}-1\right]}{e^{\left(\frac{(-2-2 \xi)}{\varepsilon}\right)}-e^{\left(\frac{(2-2 \xi)}{\varepsilon}\right)},} & -1 \leq \xi \leq x \leq 1 .\end{cases}$

and

$R_{m}\left(\vec{u}_{m-1}\right)=\varepsilon u_{m-1}^{\prime \prime}+2 u_{m-1}+u_{m-1}+0.3$,

Now, the solution of the $m t h$-order deformation equation (4.7) for $m \geq 1$ is

$u_{m}(x)=x_{m} y_{m-1}(x)+h \int_{0}^{x} \int_{0}^{x} G(x, \xi) R_{m}\left(\vec{u}_{m-1}\right) d x d x$,

From Eq. (4.9) the first iterations are given by:

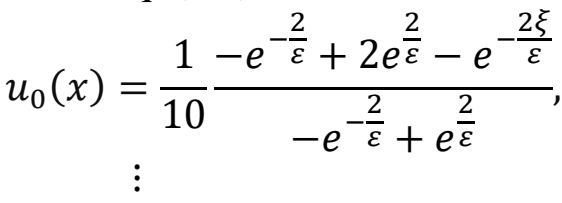

etc., in the same manner the rest of the iterations. Thus, the solution in a series form when $\boldsymbol{h}=-\mathbf{1}$ is

$u(x)=u_{0}(x)+\sum_{m=1}^{6} u_{m}(x)$.

In Tables 13-15 display a comparison of the numerical results applying the HAM ( $n=$ 6), iteration of the integral equation (IIE) (4.9) and the numerical solution of (4.9) with the Simpson rule (SIMP) with the exact solution (4.3). Twenty points have been used in the Simpson rule. In Tables 16-18 we list the maximum absolute error (MAE), the maximum relative error (MRE) and the maximum residual error (MRR) obtained by the HAM with the exact solution (4.3) on the interval $[-1,1]$. The estimated orders of convergence (EOC) and the global estimated orders of convergence (GEOC) within the interval $[-1,1]$ are given in Table 19. Fig. 21 represents both the exact solution $u_{\text {Exact }}(x)$ and our approximation by HAM $(\boldsymbol{n}=\mathbf{5})$ in the interval $-1 \leq x \leq 1$. We note that the excellent adjustment is appreciated in the turning point.

Table 13. Numerical results for $\varepsilon=\frac{1}{10}$.

\begin{tabular}{ccccc}
\hline $\boldsymbol{x}$ & $\boldsymbol{u}_{\text {Exact }}(\boldsymbol{x})$ & HAM & IIE & SIMP \\
\hline-1.0 & 0.100000000 & 0.099999999 & 0.100000000 & 0.099999999 \\
-0.8 & 0.939094689 & 0.938148844 & 0.938931367 & 0.938931362 \\
-0.6 & 0.836052132 & 0.835518724 & 0.835966278 & 0.835966278 \\
-0.4 & 0.725600744 & 0.725333540 & 0.725561168 & 0.725561168 \\
-0.2 & 0.625568681 & 0.625446098 & 0.625552096 & 0.625552097 \\
0.0 & 0.535286779 & 0.535236389 & 0.535280594 & 0.535280595 \\
0.2 & 0.453811030 & 0.453793135 & 0.453809051 & 0.453809051 \\
0.4 & 0.380282606 & 0.380277429 & 0.380282093 & 0.380282093 \\
0.6 & 0.313926310 & 0.313925213 & 0.313926213 & 0.313926213
\end{tabular}




\begin{tabular}{ccccc}
\hline 0.8 & 0.254042557 & 0.254042424 & 0.254042546 & 0.254042546 \\
1.0 & 0.199999999 & 0.200000000 & 0.200000000 & 0.200000000 \\
\hline \multicolumn{5}{c}{ Table 14. Numerical results for $\frac{1}{100}$} \\
\hline $\boldsymbol{x}$ & $\boldsymbol{u}_{\text {Exact }}(\boldsymbol{x})$ & HAM & IIE & SIMP \\
\hline-1.0 & 0.100000000 & 0.100000000 & 0.100000000 & 0.100000000 \\
-0.8 & 0.932585678 & 0.932124387 & 0.932525421 & 0.932525435 \\
-0.6 & 0.815009452 & 0.814783133 & 0.814983009 & 0.814983106 \\
-0.4 & 0.708648811 & 0.708547469 & 0.708638374 & 0.708638556 \\
-0.2 & 0.612433901 & 0.612393618 & 0.612430312 & 0.612430502 \\
0.0 & 0.525396921 & 0.525383301 & 0.525395898 & 0.525396033 \\
0.2 & 0.446662391 & 0.446658741 & 0.446662167 & 0.446662236 \\
0.4 & 0.375438339 & 0.375437658 & 0.375438307 & 0.375438330 \\
0.6 & 0.311008343 & 0.311008276 & 0.311008341 & 0.311008345 \\
0.8 & 0.252724317 & 0.252724316 & 0.252724317 & 0.252724318 \\
1.0 & 0.200000000 & 0.200000000 & 0.199999999 & 0.199999999 \\
\hline
\end{tabular}

Table 15. Numerical results for $\varepsilon=\frac{1}{200}$

\begin{tabular}{ccccc}
\hline $\boldsymbol{x}$ & $\boldsymbol{u}_{\text {Exact }}(\boldsymbol{x})$ & HAM & IIE & SIMP \\
\hline-1.0 & 0.099999999 & 0.099999999 & 0.099999999 & 0.099999999 \\
-0.8 & 0.931189334 & 0.930747883 & 0.931132619 & 0.931132705 \\
-0.6 & 0.813886584 & 0.813671108 & 0.813861875 & 0.813862264 \\
-0.4 & 0.707759967 & 0.707664115 & 0.707750304 & 0.707750828 \\
-0.2 & 0.611744665 & 0.611706899 & 0.611741384 & 0.611741815 \\
0.0 & 0.524877314 & 0.524864701 & 0.524876394 & 0.524876651 \\
0.2 & 0.446286334 & 0.446283015 & 0.446286138 & 0.446286251 \\
0.4 & 0.375183184 & 0.375182584 & 0.375183157 & 0.375183191 \\
0.6 & 0.310854456 & 0.310854401 & 0.310854454 & 0.310854460 \\
0.8 & 0.252654709 & 0.252654708 & 0.252654709 & 0.252654709 \\
1.0 & 0.199999999 & 0.199999999 & 0.200000000 & 0.200000000 \\
\hline
\end{tabular}

Table 16. MAE, MRE and MRR for $\varepsilon=\frac{1}{10}$.

\begin{tabular}{cccc}
\hline $\boldsymbol{m}$ & MAE & MRE & MRR \\
\hline 3 & $9.574 \mathrm{E}-02$ & $1.042 \mathrm{E}-01$ & $2.105 \mathrm{E}-01$ \\
4 & $2.441 \mathrm{E}-02$ & $2.693 \mathrm{E}-02$ & $7.133 \mathrm{E}-02$ \\
5 & $5.327 \mathrm{E}-03$ & $5.948 \mathrm{E}-03$ & $1.908 \mathrm{E}-02$ \\
6 & $1.028 \mathrm{E}-03$ & $1.164 \mathrm{E}-03$ & $4.299 \mathrm{E}-03$ \\
\hline
\end{tabular}

Table 17. MAE, MRE and MRR for $\frac{1}{100}$

\begin{tabular}{cccc}
\hline $\boldsymbol{m}$ & MAE & MRE & MRR \\
\hline 3 & $1.025 \mathrm{E}-01$ & $9.959 \mathrm{E}-02$ & $2.389 \mathrm{E}-01$
\end{tabular}




\begin{tabular}{cccc}
\hline 4 & $2.403 \mathrm{E}-02$ & $2.335 \mathrm{E}-02$ & $7.843 \mathrm{E}-02$ \\
5 & $4.613 \mathrm{E}-03$ & $4.484 \mathrm{E}-03$ & $1.941 \mathrm{E}-02$ \\
6 & $6.403 \mathrm{E}-04$ & $7.288 \mathrm{E}-04$ & $3.864 \mathrm{E}-03$ \\
\hline \multicolumn{4}{c}{ Table 18. MAE, MRE and MRR for $\varepsilon=\frac{1}{200}$} \\
\hline $\boldsymbol{m}$ & MAE & MRE & MRR \\
\hline 3 & $9.318 \mathrm{E}-02$ & $9.875 \mathrm{E}-02$ & $2.383 \mathrm{E}-01$ \\
4 & $2.360 \mathrm{E}-02$ & $2.298 \mathrm{E}-02$ & $7.783 \mathrm{E}-02$ \\
5 & $4.486 \mathrm{E}-03$ & $4.367 \mathrm{E}-03$ & $1.912 \mathrm{E}-02$ \\
6 & $7.198 \mathrm{E}-04$ & $7.008 \mathrm{E}-04$ & $3.766 \mathrm{E}-03$ \\
\hline
\end{tabular}

Table 19. EOC and GEOC

\begin{tabular}{cccc}
\hline$\varepsilon$ & $x=-0.75$ & $x=-0.95$ & GEOC \\
\hline$\frac{1}{10}$ & 1.1127 & 1.1201 & 1.0983 \\
$\frac{1}{100}$ & 1.1326 & 1.1405 & 1.1185 \\
$\frac{1}{200}$ & 1.1337 & 1.1415 & 1.1197 \\
\hline
\end{tabular}

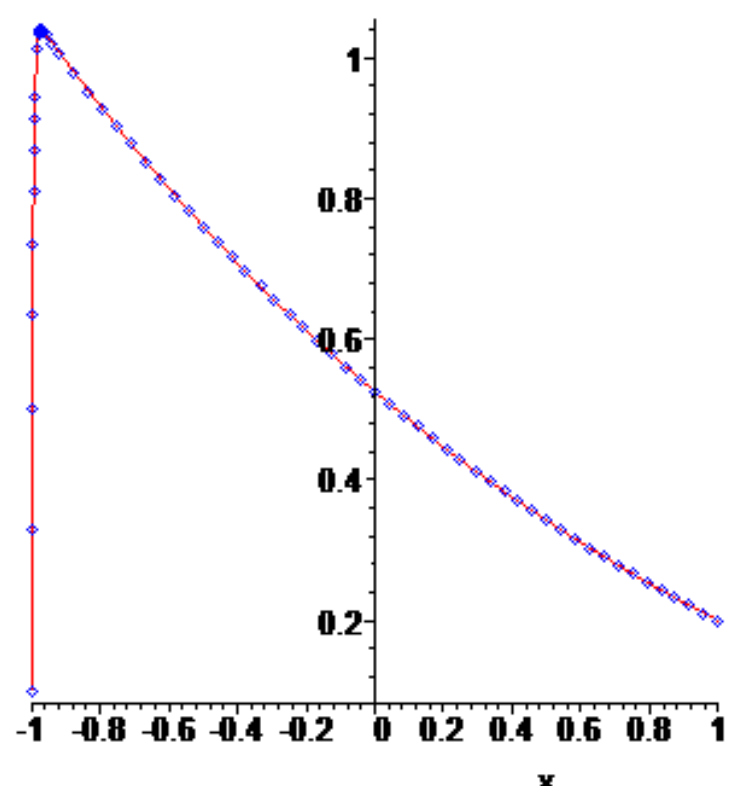

Fig. 21. Continuous line: $u_{\text {Exact }}(x),+: H A M, \varepsilon=\frac{1}{100}$

\section{Conclusions}

In this work, the HAM has been successfully applied to solve BVPs of second order with turning point of large and small parameter respectively.

The HAM has been used directly without any need for transformation formulae or restrictive assumptions. The solution process by the HAM is compatible with the method in the literature providing analytical approximation such as ADM. The results obtained in all cases demonstrate the reliability and the efficiency of this method is better than the ADM. 


\section{REFERENCES}

[1] Al-Hayani W. and Casasús L., (2005), The Adomian decomposition method in turning point problems, Journal of Computational and Applied Mathematics 177, 187-203.

[2] Ji-Huan He, (2003), Homotopy perturbation method: a new nonlinear analytical technique. Appl. Math. Comput. 135 (1), 73-79.

[3] Liao S. J. (1999), An explicit totally analytic approximation of Blasius' viscous flow problems, International Journal of Non-Linear Mechanics, 34 (4), 759-778.

[4] Liao S. J., (1992), The proposed homotopy analysis technique for the solution of nonlinear problems, (Doctoral dissertation, Ph.D. Thesis, Shanghai Jiao Tong University).

[5] Liao S. J., (2003), Beyond perturbation: introduction to the homotopy analysis method. CRC press.

[6] Linss T. and R. Vulanovic R., (2002), Uniform methods for semilinear problems with attractive boundary turning points, Proc. Appl. Math. Mech. 1, 518-519.

[7] Linss T. and Vulanovic R., (2001), Uniform methods for semilinear problems with an attractive boundary turning point, Novi Sad J. Math. 31, 99-114.

[8] Manohar R. P. and Ngai T. Y., (1991), Automated allocation of mesh points for stiff two-point boundary value problems, Computers Math. Applic. 22 (3), 7579.

[9] Nayfeh A. H., (1973), Perturbation Methods (John Wiley \& Sons, Inc.).

[10] Shao S. and Chin R. C. Y., (1996), An Asymptotic-Numerical Method for a Time-Dependent Singularly Perturbed System with Turning Points, Numerical Methods for Partial Differential Equations, 12, 441-460. 